\title{
Situación de las Unidades de Cuidados Intensivos Neonatales (UCIN) del sector público en Paraguay (periodo agosto 2013 - agosto 2014)
}

\author{
Neonatal Intensive Care Units (NICU) status in Paraguayan \\ Public Health Facilities in Paraguay during the time period \\ from August 2013 to August 2014
}

\author{
Ezequiel González ${ }^{(1)}$, Gustavo González ${ }^{(2)}$
}

\section{RESUMEN}

Introducción: Se estima que el 10\% de los recién nacidos requieren cuidado especial y el $1 \%$ requerirá atención en Unidades de Cuidados Intensivos Neonatales (UCIN). Objetivo: Describir la situación de las UCIN del sector público del Paraguay, entre agosto 2013 a agosto 2014. Material y Método: Estudio observacional, descriptivo, transversal. Participaron las UCIN del Hospital de Clínicas de la Universidad Nacional de Asunción y del Ministerio de Salud Pública y Bienestar Social de capital e interior. Se evaluaron: número de UCIN, distribución geográfica, recursos edilicios, infraestructura física, servicios de apoyo, recursos humanos y establecimiento de residencia médica. Resultados: El sector público tiene 10 UCIN distribuidas: 4 Asunción, 4 Departamento Central y 2 Interior del país. El total de camas es 58 en intensivos y 65 en intermedios. La evaluación arrojó la falta de, detectores de humo en $60 \%$ de los casos, extinguidores de incendio y salidas de emergencia en el $70 \%$ de los servicios, depósito de insumos en el $20 \%$ de las unidades, servicios de radiología, laboratorio y banco de sangre las 24 horas en un $10 \%$ de los servicios. En relación a los recursos humanos, la cantidad de médicos que trabajan en las UCIN son 154 personas; cuentan con la especialidad 57 profesionales. Existe sistema de residencia en el 30\% de las UCIN. Conclusión: Luego de la evaluación del número de UCIN, la distribución geográfica, los recursos edilicios, la infraestructura física, los servicios de apoyo, los recursos humanos y capacitación, requieren una urgente atención, debido al déficit que se encontraron en cada uno de los aspectos evaluado.

Palabras clave: Unidades de Cuidados Intensivos Neonatales, sector público, Paraguay.

\section{ABSTRACT}

Introduction: It is estimated that $10 \%$ of newborns require special care at delivery and $1 \%$ of newborns require attention in Neonatal Intensive Care Units (NICU). Objective: Describe the situation of NICU in the Public Health Sector in Paraguay between August 2013 and August 2014. Materials and Methods: This was an observational, descriptive, cross-sectional study. We reviewed all neonatal intensive care units at the Hospital of the National University of Asuncion Hospital and all hospitals in the Ministry of Public Health and Social Welfare Health network, both in the national capital area and the country's interior. We evaluated: number of NICUs, geographic distribution, equipment support, physical infrastructure, support services, human resources and having a medical residency associated with the unit. Results: The Public Health Sector has 10 NICU distributed as follows: 4 in Asunción (capital city), 4 in the Central Department, 2 in the country's interior. The total beds are: 58 beds for intensive-level care and 65 beds for intermediate-level care. The evaluation showed a lack of smoke detectors in $60 \%$ of cases, fire extinguishers and emergency exits in $70 \%$ of services, supplies stations in $20 \%$ of the units, radiology, laboratory and 24 hour Blood-bank services in $10 \%$ of units. Regarding human resources, the number of doctors working in the NICU were 154 physicians, of which 57 are certified in a medical specialty. There were residency programs in $30 \%$ of the NICU. Conclusion: After evaluating the number of NICU, geographic distribution, equipment resources, physical infrastructure, support services, human resources and training, urgent attention is needed to correct the deficits that were found in each of the evaluated aspects.

Keywords: Neonatal Intensive Care Units, public sector, Paraguay.

\footnotetext{
1. Auditor médico del Ministerio de Salud Pública y Bienestar Social. Asunción, Paraguay.

2. Director de Terapias y Urgencias del Ministerio de Salud Pública y Bienestar Social. Asunción, Paraguay.

Correspondencia: Dr. Ezequiel González. E-mail: raulexzequiel@hotmail.es

Recibido: 27/11/2014; Aceptado: 10/06/2015.

http://dx.doi.org/10.18004/ped.2015.agosto.134-138

Los autores declaran que no existen conflictos de interés en el presente estudio.
}

$134 \quad$ Pediatr. (Asunción), Vol. 42; № 2; Agosto 2015; pág. 134 - 138 


\section{INTRODUCCIÓN}

Según estadísticas de la Organización Panamericana de la Salud, el 10\% de los recién nacidos requieren algún cuidado especial y el 1\% deben recibir atención especializada en unidades de cuidados intensivos ${ }^{(1)}$.

El impacto que tienen los cuidados intensivos en los recién nacidos graves en la sobrevida de estos pacientes, está demostrada por diversos estudios ${ }^{(2)}$. Pero esta atención implica altos costos, pues se requiere de tecnología, condiciones edilicias especiales y recursos humanos especializados. Para subsanar estos problemas se establecieron los sistemas de regionalización de atención perinatal ${ }^{(3)}$. La estrategia de la regionalización de la asistencia perinatal ha supuesto una significativa reducción de la mortalidad y morbilidad perinatal. Su planificación se ha adaptado al modelo de organización sanitaria establecido en cada región, según factores políticos, económicos, geográficos, demográficos e incluso éticos ${ }^{(4)}$.

Las UCIN es el nivel más alto de asistencia, y el requerimiento de camas calculado es de una cama de intensivo y 4 camas de intermedio por cada 1000 nacimientos ${ }^{(1,5)}$. En los últimos 20 años la tasa registrada de mortalidad infantil en Paraguay se ha reducido en más de la mitad, pasando de 30,4 en 1990 a 15,2 en $2011^{(6)}$. Sin embargo, la mortalidad neonatal, apenas ha descendido de 14,4 por cada 1000 nacidos vivos en 1990 a 11,2 por 1000 nacidos vivos en 2011, y es una de las más altas de la región. En Paraguay de cada 100 niños y adolescentes que fallecen, 41 mueren antes de los 28 días de vida ${ }^{(6)}$.

Según informes del censo nacional de salud del Ministerio de Salud Pública y Bienestar Social, la cantidad de partos es de 105.000 nacimientos al año ${ }^{(7)}$. Estos se encuentran distribuidos según departamentos de la siguiente manera: I Concep-ción: 4.297, II San Pedro: 6.513, III Cordillera: 4.166, IV Guairá: 2.936, V Caaguazú: 7.690 VI Caazapá: 2.450, VII Itapúa: 7.365, VIII Misiones: 1.851, IX Paraguarí: 3.103, X Alto Paraná: 13.957, XI Central: 31.359, XII Ñeembucú: 893, XIII Amambay: 2.720, XIV Canindeyú: 3.111, XV Presidente Hayes: 2.024, XVI Boquerón: 1.420, XVII Alto Paraguay: 241 y XVIII Asunción: 9.480 de nacimientos registrados.

El objetivo de este trabajo es describir la situación de las Unidades de Cuidados Intensivos Neonatales del sector público del Paraguay, en el periodo que va de agosto de 2013 a agosto de 2014.

\section{MATERIAL Y MÉTODO}

Estudio observacional, descriptivo, de corte transversal. Participaron las UCIN del Hospital de Clínicas de la Universidad Nacional de Asunción y del Ministerio de Salud Pública y Bienestar Social de capital e interior. Se evaluaron: número de UCIN, distribución geográfica, recursos edilicios, infraestructura física, servicios de apoyo, recursos humanos y establecimiento de residencia médica. Para la evaluación de los recursos edilicios, la infraestructura física, el área social y enfermería se tomó las directrices para habilitación y funciona-miento de los servicios de terapia intensiva adultos, pediátricos y neonatales de Mercosur GMC/RES № 65/06 ${ }^{(8)}$. Para el reclutamiento de datos se confec-cionó una ficha técnica, con los datos requeridos, que fueron completados por el personal participante de este estudio. Para la recolección de los datos sobre los recursos edilicios e infraestructura se realizó una visita a cada servicio y se procedió a completar la ficha técnica previamente confeccionada. Para la evaluación de la cantidad de especialistas, se consultó a la página web de la Sociedad Paraguaya de Pediatría y se cotejó con los datos aportados por la ficha técnica, en virtud a un convenio vigente entre el Ministerio de Salud Pública y Bienestar Social y el Círculo Paraguayo de Médicos, a través de las sociedades científicas, que delega en estas últimas, el reconocimiento y la acreditación de las carreras de especialidades médicas ${ }^{(9)}$.

\section{RESULTADOS}

En el sistema de salud pública del Paraguay existen 10 unidades de cuidados intensivos neonatales (9 pertenecientes al Ministerio de Salud Pública y Bienestar Social y una unidad perteneciente a la Universidad Nacional de Asunción). El total de camas para cuidados intensivos es de 58 y el número camas para cuidados intermedios es 65 . En la tabla 1 se observa la distribución geográfica y en la tabla 2 se describen la cantidad de camas existentes en cada hospital. 
Tabla 1. Distribución de camas en cuidados intensivos e intermedios neonatales según localidad.

\begin{tabular}{lccccc}
\hline & UCIN & $\begin{array}{c}\mathbf{N}^{\circ} \text { de camas } \\
\text { en intensivos }\end{array}$ & $\begin{array}{c}\text { \% de } \\
\text { camas }\end{array}$ & $\begin{array}{c}\mathbf{N}^{\circ} \text { de camas } \\
\text { en intermedios }\end{array}$ & $\begin{array}{c}\text { \% de } \\
\text { camas }\end{array}$ \\
\hline Asunción & 4 & 25 & $43 \%$ & 29 & $45 \%$ \\
Central & 4 & 26 & $45 \%$ & 28 & $43 \%$ \\
Interior & 2 & 7 & $12 \%$ & 8 & $12 \%$ \\
Total & 10 & 58 & $100 \%$ & 65 & $100 \%$ \\
\hline
\end{tabular}

Tabla 2. Número de camas en unidades de cuidados intensivos e intermedios neonatales en hospitales públicos de Paraguay.

\begin{tabular}{lcc}
\hline Hospital & UCIN & Intermedios \\
\hline Hospital Materno Infantil de Trinidad & 6 & 3 \\
\hline Hospital Materno Infantil Reina Sofía, Cruz Roja Paraguaya & 3 & 10 \\
\hline Hospital Materno Infantil Barrio San Pablo & 12 & 10 \\
\hline Hospital Materno Infantil Barrio Obrero & 4 & 6 \\
\hline Hospital de Clínicas. UNA & 10 & 10 \\
\hline Hospital General Nacional Itagua & 10 & 6 \\
\hline Hospital Regional de Luque & 2 & 6 \\
Hospital Materno Infantil de San Lorenzo & 4 & 6 \\
\hline Hospital Regional de Coronel Oviedo & 3 & 6 \\
\hline Hospital Regional de Ciudad del Este & 4 & 2 \\
\hline TOTAL & 58 & 65 \\
\hline
\end{tabular}

El resultado de la evaluación de los recursos edilicios e infraestructura verificado en las visitas a cada servicio se muestra en las tablas 3,4 y 5 .

Tabla 3. Resultados de la evaluación de recursos edilicios de UCIN.

\begin{tabular}{ll}
\hline Variables & Resultados \\
\hline Paredes lavables & $100 \%$ de los servicios \\
\hline Pisos sin solución de continuidad & $80 \%$ de los servicios \\
\hline Unión Piso/Pared/Techo redondeada & $35 \%$ de los servicios \\
\hline Iluminación natural & $60 \%$ de los servicios \\
\hline Generador eléctrico & $60 \%$ de los servicios \\
\hline Bomba de agua & $20 \%$ de los servicios \\
\hline Detector de humo & $40 \%$ de los servicios \\
\hline Extinguidor & $30 \%$ de los servicios \\
\hline Salida de emergencia & $30 \%$ de los servicios \\
\hline
\end{tabular}

Tabla 4. Resultados de la evaluación de la infraestructura física exclusivamente del área de cuidados intensivos neonatales.

\begin{tabular}{lcc}
\hline & Total & Promedio cama \\
\hline Camas & 58 & 5,8 camas por hospital \\
Superficie Cama & $510 \mathrm{mts} 2$ & $8,8 \pm 2 \mathrm{mts} 2$ \\
\hline Tomas 02 & 135 & $13,5 \times$ unidad \\
Tomas de aire medicinal & 68 & $6,8 \times$ unidad \\
Tomas de vacío & 116 & $11,6 \times$ unidad \\
Lavamanos & 20 & $1 \times 3$ camas \\
Toma corriente & 588 & $10 \times$ camas \\
\hline
\end{tabular}

Tabla 5. Resultados de la evaluación del área social y área de enfermería.

\begin{tabular}{ll}
\hline Variable & Resultados \\
\hline Oficina para médicos & $60 \%$ de los servicios \\
\hline Sala de conferencia & $80 \%$ de los servicios \\
\hline Estar médico aprobado & $70 \%$ de los servicios \\
\hline Albergue para familiares & $70 \%$ de los servicios \\
Vestuario de enfermería aprobado & $60 \%$ de los servicios \\
\hline Área limpia de enfermería aprobado & $70 \%$ de los servicios \\
\hline Área sucia de enfermería aprobado & $60 \%$ de los servicios \\
\hline Depósito para insumos & $80 \%$ de los servicios \\
\hline
\end{tabular}

En relación a la cantidad de especialistas se encontró en total 57 neonatólogos trabajando en UCIN. De 10 coordinadores de UCIN, el $60 \%$ son neonatólogos; de 29 médicos diaristas el $51 \%$ son neonatólogos y de 115 médicos de guardia, 5\% son especialistas neonatólogos. El sistema de capacitación en residencias médicas en neonatología se desarrolla en 3 hospitales representando el $30 \%$ de los casos.

En cuanto a los servicios de apoyo se encontró que cuentan con servicios de laboratorio de análisis clínicos, radiología y banco de sangre en el 90\% de las unidades; servicio de farmacia las 24 horas en un $70 \%$ de los casos, cuentan con cirujano $40 \%$, con kinesiólogo $20 \%$, con psicólogo $50 \%$, infectólogo $30 \%$, Comité de Bioética $10 \%$ y trabajador social en el $90 \%$ de los casos.

\section{DISCUSIÓN}

Los resultados de este trabajo, permitieron evaluar a las 10 unidades de cuidados intensivos del sector público del país. Informes del Censo de Salud del 2013 del Ministerio de Salud Pública y Bienestar Social estiman que la cantidad de partos al año es de 105.000 nacimientos $^{(7)}$. Las estadísticas de la Organización Panamericana de Salud estiman que el 1\% deben recibir atención en UCIN. El cálculo de las camas propuesto por el Dr. Augusto Sola ${ }^{(5)}$ de una cama de cuidados intensivos y 4 camas de cuidados intermedios por cada 1000 nacimientos nos permite estimar que se necesita aproximadamente 100 camas de terapia intensiva y 400 camas de intermedia ${ }^{(5)}$. Los datos recogidos muestran un déficit en el número requerido de camas de terapia intensiva e intermedia. Además muestra una mala proporción de camas Intensiva versus Intermedia, lo cual dificulta el buen funcionamiento de los respectivos servicios de $\mathrm{UCIN}^{(5)}$.

El alto costo que implica la atención en las UCIN, llevó al desarrollo de estrategias de regionalización de la asistencia perinatal, teniendo en cuenta el área 
geográfica y ha supuesto una significativa reducción de la mortalidad y morbilidad perinatal ${ }^{(3-5)}$. Es así que al distribuir la región oriental, en área norte (integrada por Concepción, San Pedro, Amambay) se estiman 13530 nacimientos, que no cuentan con servicios de UCIN. En el área este (integrada por Caazapá, Canindeyú, y Alto Paraná), se estiman 19127 nacimientos, está área cuenta en Cuidad del Este con 4 camas de cuidados intensivos y 2 intermedios. En el área centro (integrada por Caaguazú, Cordillera, Guiara y Paraguarí) se estiman 17835 nacimientos, se cuenta con UCIN en la Cuidad de Coronel Oviedo con 3 camas intensivas y 6 intermedias. En el área sur (integrada por Itapuá, Ñeembucú y Misiones), se estiman 10109 nacimientos que no cuentan con UCIN. En cuanto al área metropolitana (integrada por Asunción y el Departamento Central) que registra el mayor número de nacimiento en el país, (aproximadamente 41.000/año) lo cual requiere de 41 camas intensivas y entre 75 a 80 camas de intermedia, actualmente la distribución de camas correspondientes a Asunción y Central es de 51 camas intensivas y 57 camas de intermedia. Para el área oeste formada por la región occidental integrada por los departamentos de Presidente Hayes, Boquerón y Alto Paraguay se estima 3685 nacimientos, esta área no cuenta con servicios de UCIN.

Según estos datos la distribución de camas necesaria para cada área sería (Tabla 6).

Tabla 6. Distribución de camas necesarias según aéreas geográficas.

\begin{tabular}{lccc}
\hline Área & Nacimiento & $\begin{array}{c}\text { Camas de UCIN } \\
\text { necesarias }\end{array}$ & $\begin{array}{c}\text { Camas de } \\
\text { Intermedia necesarias }\end{array}$ \\
\hline Norte & 13530 & 13 & 52 \\
Este & 19127 & 19 & 76 \\
Centro & 17835 & 17 & 68 \\
Sur & 10109 & 10 & 40 \\
Metropolitana & 41.000 & 41 & 84 \\
Oeste & 3685 & 3 & 12 \\
\hline
\end{tabular}

La evaluación de los recursos edilicios, infraestructura física, área social y enfermería realizado según las directrices para habilitación y funcionamiento de los servicios de terapia intensiva adulto, pediátrica y neonatal en el Mercosur ${ }^{(8)}$, que establece: 1) los Servicios de Terapia Intensiva deben disponer de infraestructura física con ambientes e instalaciones necesarios para la asistencia y la realización de los procedimientos con seguridad y calidad; 2) la habitación colectiva para internación de los pacientes neonatos con área mínima de $6,5 \mathrm{~m}^{2}$ por incubadora o cuna; 3) puesto de enfermería que permita la observación visual directa o electrónica de las camas, 01(uno) para cada 10 (diez) camas; 4) área para prescripción médica; 5) sala de utilidades o material sucio dotada de pileta con ducha manual para lavado y pileta con válvula de descarga; 6) depósito de material de limpieza dotado de recipiente para lavado; 7) sala administrativa; 8) sala de descanso del equipo de salud, con baño compuesto de lavatorio, inodoro y ducha; 9) área de estar y colaciones para el equipo de salud; 10) vestuarios para personal (masculino y femenino) dotado de lavatorio e inodoro; 11) lavatorio para uso del equipo de asistencia, 01(uno) a cada 05 (cinco) camas; 12) paredes, pisos y techos revestidos de material liso, resistente al lavado y al uso de desinfectante; 13) los pisos deben ser resistentes a los abrasivos y a los impactos a los que serán sometidos.

Los Servicios de Terapia Intensiva deben poseer instalaciones generales que comprendan las siguientes características: 5.3.1 sistema de energía eléctrica alternativo de emergencia para alimentación de los equipos de soporte vital y de circuitos de iluminación de emergencia; 5.3.2 circuitos de iluminación distintos de los circuitos eléctricos especiales, desde la fuente de entrada, con el fin de evitar interferencias electromagnéticas en los equipos e instalaciones; 5.3 .3 sistema de abastecimiento de gas medicinal centralizado, con 02 (dos) puntos de oxígeno, 01 (uno) de vacío y 02 (dos) de aire medicinal por cama; 5.3.4 sistema de señalización luminosa interconectando cada cama al puesto de enfermería. 5.3.5 sistema de climatización que posibilite confort térmico al paciente y el mantenimiento de la calidad del aire interior, con dos tipos de filtro (filtros clase G3+F7), para los Servicios de Terapia Intensiva Adulto y Pediátrica; 5.3.5.1 la habitación de aislamiento de los Servicios de Terapia Intensiva Adulto y Pediátrica debe poseer sistema de ventilación/extracción que permita $100 \%$ de renovación de aire interior; 5.3.6 sistema de climatización que posibilite confort térmico al paciente y el mantenimiento de la calidad del aire interior, con un tipo de filtro (clase G4), para los Servicios de Terapia Intensiva Neonatal.

Al analizar los recursos edilicios, basado en las directrices del Mercosur, encontramos falencias que requieren urgente atención, como los sistemas de seguridad contra incendios, detector de humo, extinguidores de fuego y salidas de emergencia. Las instalaciones físicas en las que se proporciona los cuidados perinatales deben permitir que la asistencia cumpla con las necesidades fisiológicas y psicosociales 
de los neonatos, familiares y trabajadores de la salud. El estar médico y el vestidor de enfermería deben contar con los requisitos mínimos (baño, casillero, etc.). Otra grave falencia detectada fue la ausencia de depósito de insumos en algunas las unidades. En lo referente a los servicios de apoyo se encontró la necesidad de contar con cirujanos e infectólogos, servicios de radiología, laboratorio y banco de sangre las 24 horas.

En cuanto a los recursos humanos, entre las condiciones emanadas por estudiosos del tema como el Dr. Solá, para mejorar la calidad de la atención de estos pacientes se expresa la necesidad de que los jefes de servicios de neonatología, posean título habilitante en la especialidad ${ }^{(10)}$. Las UCIN deberán contar con por lo menos un neonatólogo de planta para conducir la correcta atención de los pacientes. El personal médico de planta, como así también de guardia, podrá estar integrado por pediatras con formación neonatológica ${ }^{(11)}$. En relación a estos aspectos, este trabajo encontró un déficit preocupante en cuanto a la cantidad de médicos con título de neonatólogos que trabajan en las UCIN. Así se constató que solo el 60\%

\section{REFERENCIAS}

1. Goldsmith J. Generalidades y principios sobre reanimación. En: Katlwinkeld J, editor. Reanimación neonatal. $6^{\text {ta }}$ ed. American Heart Association; 2006. p. 1-3.

2. Ruza F. Unidad de cuidados intensivos pediátricos en la asistencia pediátrica actual. En: Ruza F, editor. Tratado de cuidados intensivos pediátricos. Madrid: Ediciones Norma; 1993. p. 3-25.

3. Avary M, Richardson D. Historia y epidemiologia. En: Taeusch HW, Ballard R, editores. Tratado de neonatología de Avery. $7^{\mathrm{ma}}$ ed. Buenos Aires: Panamericana; 2000.p. 3.

4. Schnitzler E. Categorización de unidades o servicios de neonatología. Disponible en: www.itaes.org.ar/biblioteca/ 52011/ITAES-5-2011-ss-salud.pdf

5. Sola A. Niveles asistenciales y recomendaciones de mínimos para la atención neonatal. En: Sola A, editor. Cuidados especiales neonatales. $3^{\text {ra }}$ ed. Buenos Aires: Edimed;2011.p. 1787.

6. Unicef. Situación del derecho a la salud materna, infantil y adolescente en Paraguay. Asunción: Unicef; 2013. Disponible en: www.unicef.org/paraguay/spanish/ unicefinformesalud.pdf

7. Ministerio de Salud Pública y Bienestar Social. Indicadores básicos de salud del Paraguay 2013. MSP y BS/OPS. Asunción; 2013. de los coordinadores de UCIN son neonatólogos. Siendo lo más llamativo la ausencia de especialistas (neonatólogos) en las unidades de cuidados intensivos en el interior del país ${ }^{(12-14)}$. Otra de las finalidades de las UCIN está orientada hacia la formación de especialistas, encontrándose también déficit en este campo pues solo en el $30 \%$ de las unidades se pudo constatar el desarrollo de cursos de especialistas en neonatología ${ }^{(11)}$.

\section{CONCLUSIÓN}

La situación de las Unidades de Cuidados Intensivos Neonatales del sector público del Paraguay, luego de la evaluación de los recursos edilicios, la infraestructura física, los servicios de apoyo, los recursos humanos y capacitación, requieren una urgente atención, debido al déficit que se encontraron en cada uno de los aspectos evaluados, más aun, teniendo en cuenta la acuciante necesidad de mejorar la elevada tasa de mortalidad neonatal que subsiste en nuestro país.

8. Mercosur. GMC/RES № 65/06 directrices para habilitación y funcionamiento de los servicios de terapia intensiva adulto, pediátrica y neonatal. Brasilia. Mercosur; 2006. Disponible en: www.sice.oas.org/trade/mrcsrs/ resolutions/Res6506.pdf

9. Convenio de cooperación entre el Ministerio de Salud Pública y Bienestar Social y el Círculo Paraguayo de Médicos, en materia de control del ejercicio profesional de las especialidades médicas. Asunción, 20 de noviembre de 2008.

10. Cruz, H. Organización de servicio. En: Sola A, editor. Cuidados especiales neonatales. 2 da ed. Buenos Aires: Edimed;2000.p. 1771.

11. Sola A, Birbaum S. Regionalización y estadísticas vitales. En: Sola A, editor. Cuidados especiales neonatales. 2 da ed. Buenos Aires: Edimed;2000. p. 1787.

12. Doldan O. Situación de los cuidados intensivos pediátricos en el Paraguay. Pediatr (Asunción). 2002;23(2): 180-84.

13. Bernada, M. Censo nacional de pediatría intensiva y neonatal. Arch Pediatr Urug. 2012;83(4):278-85.

14. López-Herce Cid J, Sancho Pérez L, Martinón Sánchez JM. Informe de la Sociedad Española de Cuidados Intensivos pediátricos: distribución de los cuidados intensivos pediátricos en España. An Esp Pediatr. 1999;50:14-16. 